\title{
Localization of speech and non-speech sounds
}

\author{
SUMI SHIGENO and TADASU OYAMA ${ }^{1}$ \\ Department of Psychology, Faculty of Letters, University of Tokyo, Bunkyo-ku, Tokyo 113
}

The localization of speech and non-speech sounds in the horizontal plane was investigated. Four kinds of stimulus sounds were employed: synthetic vowel, pure tone, white noise, and single resonance tone. The experiment was carried out under two presentation conditions: single-tone and two-successive-tone conditions. The results indicated that the percentage of correct responses for white noise was greater than that for synthetic vowels under all conditions, and that pure tone was the most difficult to be localized among the four kinds of sounds. It was also revealed that the percentage of correct responses in the single-tone condition was smaller than in the two-successive-tone condition. These results suggest that the acoustical complexity of the stimulus sound is a critical factor in the localization of speech and non-speech sounds.

Key words: sound localization, azimuth, complexity of stimulus sound, speech sounds.

Many studies have been conducted on our ability to localize various types of sounds under dichotic listening through earphones. The results of these studies have indicated that the perception of a sound's direction rests upon three factors: interaural differences in the phase, time and intensity of the tone (Mills, 1958).

It is known, on the other hand, that the detection of a change in the spatial location for noise is more accurate than for musical tones, and even more so than for pure tones (Hornbostel, 1926). In the case of speech sounds, the investigation of the accuracy of localization performance, or the features which may enhance localization are poorly understood in human subjects. In monkey's Brown, Beecher, Moody, and Stebbins (1978) found that acuity of localization was a function of the magnitude of the change in pitch (frequency modulation) of different clear calls.

2. The authors are grateful to Mr. Naobumi Sakamoto for his help in conducting the preliminary experiment, and also to Dr. Yuko Kimura for her helpful suggestions concerning English expression.

The first author now belongs to Kitasato University, 1-15-1, Kitasato, Sagamihara-shi, Kanagawa, 228 .
In general, however, few studies have looked at the localization of speech sounds rather than the localization of pure tones and noises. The purpose of our present study is to compare the localization of speech and non-speech sounds of an actual source under the same experimental conditions to investigate the effects of both the physical characteristics of the sounds and the subjective factors involved in the localization of sounds.

\section{Method}

Subjects. Four adult subjects with normal hearing (tested with an audiometer) participated in this experiment. None of them had a past history of speech or hearing problems. Prior to testing, they were familiarized with synthetic sounds.

Apparatus. The experiment was conducted in a soundproof room. Figure 1 shows the layout of the two loudspeakers, one of which was the reference and the other was the comparison. They were placed on the radius of three meters from the subject, whose face was held in a fixed position in the sound field by a chin-andhead rest. The reference speaker was placed in front of the subject, and the comparison speaker was $2.5^{\circ}$ or $5^{\circ}$ to the 
left of the reference. These two azimuths were selected on the basis of the results of a preliminary experiment from which the minimum audible angle of pure tones was obtained $\left(2^{\circ}\right.$ to $7^{\circ}$ in the frequency range of 450 to $1200 \mathrm{~Hz}$ ). The subject's head and the centers of the speakers were positioned $1.15 \mathrm{~m}$ above the floor of the room.

Stimuli. Four kinds of stimuli were used; synthetic vowels as speech stimuli, and as non-speech stimuli pure tones, white noise, and single resonance tones. A PDP 11 computer (DEC) was used to produce the stimuli; the synthetic vowels were generated by computer simulation of a terminal analog speech synthesizer, while the single resonance tones were generated with one formant characteristic of the synthesizer, which was excited by a repetitive impulse voltage source having a fundamental frequency of $130 \mathrm{~Hz}$. All of the stimuli were read out at a sampling rate of $10 \mathrm{kHz}$ with an accuracy of 10 bits, converted into analog waveform, and recorded through a low-pass filter with a cutoff frequency of $4.5 \mathrm{kHz}$.

The synthetic vowels were typical sounds for $/ \mathrm{a} /\left(F_{1}=750 \mathrm{~Hz}, F_{2}=1200 \mathrm{~Hz}\right)$ and typical sounds for $/ o /\left(F_{1}=450 \mathrm{~Hz}, F_{2}=\right.$

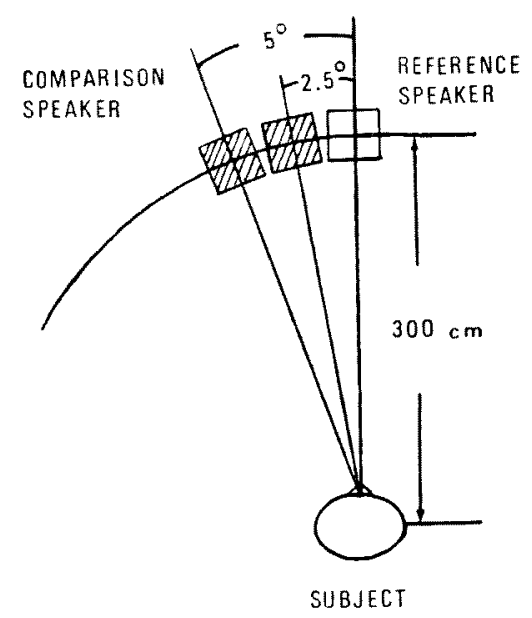

Fig. 1. Loudspeaker array for localization tests in the horizontal plane.
$750 \mathrm{~Hz}$ ). The other formant frequencies $\left(F_{3}, F_{4}\right.$, and $\left.F_{5}\right)$ were held constant at 2600,3500 , and $4500 \mathrm{~Hz}$, respectively. The bandwidths of these formants were also held constant at 60, 100, 120, 175, and $280 \mathrm{~Hz}$, for $F_{1}$ to $F_{5}$ respectively. The resonance frequencies (RF) of the single resonance tones and the frequencies (F) of the pure tones had the same value as the first or the second formant frequencies of the synthetic vowels.

Procedure. The experiment was divided into four sessions of $30 \mathrm{~min}$ to $40 \mathrm{~min}$. On any one day each subject served for two sessions with a $10 \mathrm{~min}$ inter-session rest period. During any one session the azimuth and the presentation conditions were constant.

Two presentation conditions were employed: the single-tone condition and the two-successive-tone condition. In the single-tone condition, the duration of each stimulus was $200 \mathrm{~ms}$, and nine kinds of stimulus sounds were used-pure tones at three different frequencies $(450 \mathrm{~Hz}$, $750 \mathrm{~Hz}$, and $1200 \mathrm{~Hz}$ ); white noise; single resonance tones at three different resonance frequencies $(450 \mathrm{~Hz}, 750 \mathrm{~Hz}$, and $1200 \mathrm{~Hz}$ ); and two synthetic vowels (typical sounds for $/ a /$ and typical sounds for $/ 0 /)$. In the two-successive-tone condition, on the other hand, two 100-ms tones were successively presented without interruption, and seven kinds of stimulus pairs were used-pure tones $(450-750 \mathrm{~Hz}$ and $750-450 \mathrm{~Hz}$ ), white noise, single resonance tones $(450-750 \mathrm{~Hz}$ and $750-450$ $\mathrm{Hz}$ in resonance frequency), and synthetic vowels $(/ \mathrm{a} / \mathrm{-} / \mathrm{o} /$ and $/ \mathrm{o} / \mathrm{-} / \mathrm{a} /)$.

In both presentation conditions, the interval between the first tone(s) and the second tone(s) was $1 \mathrm{~s}$, and the inter-trial interval was $3 \mathrm{~s}$. The first stimulus (or stimulus pair) was presented constantly at $54 \mathrm{~dB}(\mathrm{c})$ through the reference speaker, while the second stimulus (or stimulus pair) was presented through the reference speaker or the comparison speaker with a probability of 0.5 for each speaker. Its 
Table 1

Percentage of correct responses for speech and non-speech stimuli

\begin{tabular}{|c|c|c|c|c|c|c|}
\hline \multirow{2}{*}{$\begin{array}{l}\text { Presentation } \\
\text { condition }\end{array}$} & \multirow{2}{*}{ Azimuth } & \multirow{2}{*}{ Stimulus sounds } & \multicolumn{4}{|c|}{ Subject } \\
\hline & & & SS & YA & TI & NS \\
\hline \multirow{8}{*}{$\begin{array}{l}\text { Single-tone } \\
\text { condition }\end{array}$} & \multirow{4}{*}{$2.5^{\circ}$} & Synthetic vowels & 82 & 68 & 71 & 80 \\
\hline & & Single resonance tones & 77 & 71 & 60 & 83 \\
\hline & & White noise & 95 & 82 & 75 & 93 \\
\hline & & Pure tones & 64 & 53 & 59 & 55 \\
\hline & \multirow{4}{*}{$5^{\circ}$} & Synthetic vowels & 95 & 97 & 97 & 97 \\
\hline & & Single resonance tones & 93 & 91 & 95 & 98 \\
\hline & & White noise & 95 & 97 & 100 & 99 \\
\hline & & Pure tones & 68 & 67 & 72 & 69 \\
\hline \multirow{8}{*}{$\begin{array}{l}\text { Two-succes- } \\
\text { sive-tone } \\
\text { condition }\end{array}$} & \multirow{4}{*}{$2.5^{\circ}$} & Synthetic vowels & 89 & 74 & 88 & 87 \\
\hline & & Single resonance tones & 89 & 64 & 84 & 80 \\
\hline & & White noise & 95 & 85 & 95 & 90 \\
\hline & & Pure tones & 73 & 67 & 68 & 58 \\
\hline & \multirow{4}{*}{$5^{\circ}$} & Synthetic vowels & 95 & 98 & 98 & 95 \\
\hline & & Single resonance tones & 99 & 97 & 96 & 93 \\
\hline & & White noise & 100 & 100 & 99 & 95 \\
\hline & & Pure tones & 75 & 88 & 75 & 85 \\
\hline
\end{tabular}

amplitude was varied randomly among five levels from $48 \mathrm{~dB}(\mathrm{c})$ to $60 \mathrm{~dB}(\mathrm{c})$ in order to minimize the possibility of the subjects using slight differences in signal amplitude between the speakers as a cue.

In both presentation conditions, all of the stimuli $[(9$ kinds of stimuli $) \times(5$ intensity levels) in the single-tone condition and ( 7 kinds of stimuli) $\times(5$ intensity levels) in the two-successive-tone condition] were completely randomized within each session. Ten trials per each intensity for each stimulus were given to each subject.

For each stimulus, the subject was asked to indicate whether the direction of the stimulus sound was the same as for the reference sound or had shifted to the left. He/she was further asked to report whether the stimulus was a speech or a non-speech sound.

\section{Results}

The experimental results were very similar among the five different intensities, and consequently the fifty judgments were pooled across this variable for each stimulus condition and for each subject.

(1) Single-tone condition

Table 1 lists the results of the experiment pooled across the different frequencies. When the azimuth was $5^{\circ}$, the percentage of correct responses for the speech stimuli and that for the non-speech stimuli (except for the pure tones), reached almost $100 \%$. Accordingly, it is obvious that there are great differences between the pure tones and the other stimulus sounds $\left(\chi^{2}=112.50, p<0.001\right.$, for the synthetic vowels; $\chi^{2}=125.23, p<0.001$, for the single resonance tones; $\chi^{2}=173.4, p<0.001$, for the white noise). However, when the azimuth was $2.5^{\circ}$, some systematic differences among the stimulus sounds were obtained. The percentage of correct responses for the white noise was greater than that for both the synthetic vowels and the single resonance tones $\left(\chi^{2}=18.55, p<0.001 ; \chi^{2}=\right.$ $32.53, p<0.001$ ), and the percentage of correct responses for the pure tones was the 
lowest $\left(\chi^{2}=32,49, p<0.001\right.$, for the synthetic vowels; $\chi^{2}=29.74, p<0.001$, for the single resonance tones; $\chi^{2}=119.03, p<$ 0.001 , for the white noise).

Figure 2 shows the performance as a function of the different frequencies or the first formant (or resonance) frequencies when the azimuth was 2.5\% It also presents the percentage of judgments as speech sounds in each frequency. The results of the four subjects were pooled in this figure. In the case of $450 \mathrm{~Hz}$, there was little difference in the percentage of correct responses between the synthetic vowels and the single resonance tones $\left(\chi^{2}=0.63\right.$, $p>0.05$ ). For $750 \mathrm{~Hz}$, on the other hand, the percentage of judgments as speech sounds for the synthetic vowels was much greater than that for the single resonance tones $\left(\chi^{2}=131.8, p<0.001\right)$. In general, the percentage of correct responses for the pure tones was smaller than that for the other stimuli.

\section{(2) Two-successive-tone condition}

Table 1 and Fig. 2 show that for cach subject the percentage of correct responses for the pure tones was smaller than that for the other stimulus sounds $\left(\chi^{2}=35.03\right.$, $p<0.001$, for the synthetic vowcls; $\chi^{2}=$ $16.45, p<0.001$, for the single resonance tones; $\chi^{2}=73.53, p<0.001$, for the white noise). In general, the results in the twosuccessive-tone condition were similar to those in the single-tone condition, though the percentage of judgments as speech sound in the $450-750 \mathrm{~Hz}$ single resonance tones was very small.

(3) Comparison between the single-tone condition and the two-successive-tone condition

Table 1 and Fig. 2 show that the percentage of correct responses was a little higher in the two-successive-tone condition than in the single-tone condition $\left(\chi^{2}=10.65, p<0.01\right.$, for the synthetic vowels; $\chi^{2}=7.89, p<0.01$, for the pure
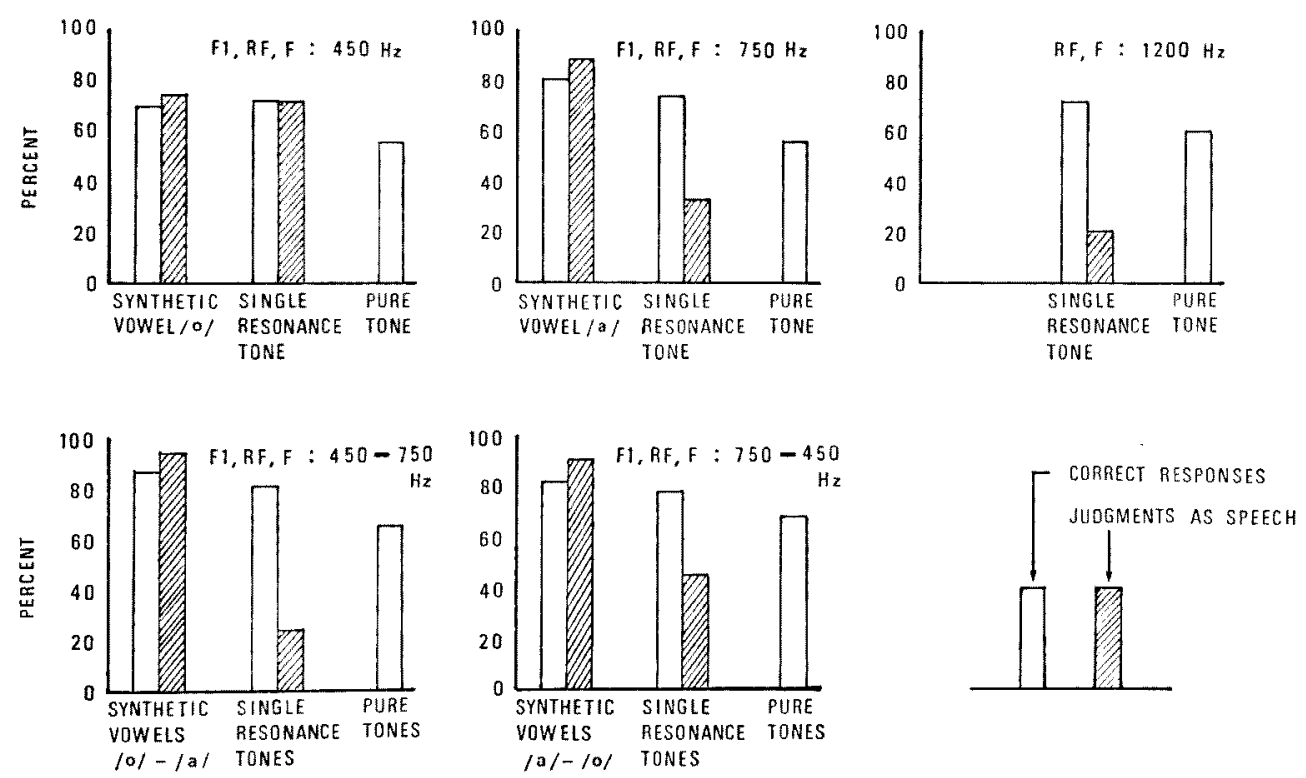

Fig. 2. Percentage of correct responses and percentage of judgments as speech sounds for the synthetic vowels, the single resonance tones, and the pure tones. The abscissa represents the three kinds of stimuli, and the ordinate represents the percentage of correct responses (blank bars) or the percentage of judgments as speech (solid bars). The upper three graphs show the results of the single-tone condition and the lower two graphs show the results of the two-successive-tone condition. The azimuth was $2.5^{\circ}$. 
Table 2

Percentage of correct responses for the stimuli judged as speech or non-speech sounds. The azimuth is $2.5^{\circ}$

\begin{tabular}{lccc}
\hline $\begin{array}{c}\text { Presentation } \\
\text { condition }\end{array}$ & $\begin{array}{c}\text { Judgment as spech } \\
\text { or non-speech }\end{array}$ & $\begin{array}{c}\text { Synthetic } \\
\text { vowels }\end{array}$ & $\begin{array}{c}\text { Single resonance } \\
\text { tones }\end{array}$ \\
\hline Single-tone condition & Speech & 81 & 78 \\
& Non-speech & 73 & 69 \\
\hline Two-successive-tone & Speech & 85 & 80 \\
condition & Non-speech & 80 & 98
\end{tabular}

tones; $\chi^{2}=37.36, p<0.001$, for the white noise; $\chi^{2}=5.59, p<0.05$, for the single resonance tones). The results also show that the single resonance (formant) tones in the single-tone condition tended to be judged as vowels very often, especially when the resonance frequency was 450 $\mathrm{Hz}$. In this experiment the resonance frequencies of the single resonance tones were identical to the first or the second formant frequencies of the synthetic vowels. Single resonance tones are usually perceived to be very similar to that of vowels when its resonance frequencies are identical to the first formant frequencies of the vowels. Here the results were not unexpected. However, in the two-successive-tone condition, the single resonance tones were not so frequently judged as vowels, and the subjects could perceive the difference between the single resonance tones and the synthetic vowels more clearly.

\section{Discussion}

The present experimental results show that complex tones such as single resonance tones and synthetic vowels, or noncyclic tones such as white noise, can be localized more accurately than pure tones. The results also show that the localization of acoustical stimuli is influenced by the physical characteristics of the stimuli rather than by subjective factors.

The present data have several implications for the localizations of speech and non-speech sounds. Though the percentage of judgments of the synthetic vowels as speech sounds was much greater than that for the single resonance tones (which have only one formant characteristic of the synthetic vowels), the percentage of correct responses in localization for the synthetic vowels was only slightly greater than that for the single resonance tones (in both the single-tone and the two-successive-tone conditions). Furthermore, the percentage of correct responses for the white noise was greater than that for the synthetic vowels.

Table 2 shows more precisely the percentage of correct responses for the stimuli judged as speech or non-speech sounds pooled across different resonance frequencies and across subjects. This table shows that the percentage of correct responses for the stimuli was not significantly influenced by whether they were judged as speech or non-speech sounds. Therefore, the critical factor for localization is neither the recognition of the stimulus sounds as speech or non-speech nor the identification of their content, but rather their acoustic complexity. In addition, the results showing that the percentage of correct responses was greater in the twosuccessive-tone condition than in the single-tone condition also support the prediction, because it is possible to say that the acoustical complexity in the twosuccessive-tone condition was greater than that in the single-tone condition. The results of Brown et al. (1978) with monkeys 
also indicated the importance of the acoustical complexity in localization.

Another possible explanation for the superiority in localization performance of the complex sounds obtained in this cxperiment is that they also contain high frequency components. However, data obtained by Mills (1958) rule out this possibility-poorer performance was obtained for high frequency pure tones than for the 450-1 $200 \mathrm{~Hz}$ tones.

In conclusion, the results of the present experiment reveal that the accuracy of localization of acoustic stimuli is determined mainly by the complexity of the stimulus sound.

\section{References}

Brown, C. H., Beecher, M. D., Moody, D. B., \& Stebbins, W. C. 1978 Localization of primate calls by old world monkeys. Science, 201, 753754.

Hornbostel, E. M. 1926 Das räumliche Hören. In A. Bethe, G. v. Bergmann, G. Embden, \& A. Ellinger (Eds.), Handbuch der normalen und pathologischen physiologie. Vol. 11. Receptionsorgane. I. Berlin: Verlag von Julius Springer. Pp. 602618.

Mills, A.W. 1958 On the minimum audible angle. Journal of the Acoustical Society of America, 30, 237-246.

(Reccived Aug. 31, 1982; accepted May 14, 1983) 